\title{
UNIQUENESS OF MEROMORPHIC FUNCTIONS CONCERNING DIFFERENTIAL POLYNOMIALS
}

\author{
SUBHAS S. BHOOSNURMATH ${ }^{1}$, VEENA L. PUJARI ${ }^{2}$ AND ANUPAMA J. PATIL ${ }^{3}$
}

\begin{abstract}
In this paper, we present a different and very simple technique to handle various uniqueness problems involving three small entire functions. It also gives a new additional insight into such problems.
\end{abstract}

\section{Introduction and the Main Results}

In this paper, the term 'meromorphic' will always mean meromorphic function in the whole complex plane $\mathbb{C}$. Let $f(z)$ be a non-constant meromorphic function.We shall use the following standard notations of value distribution theory $T(r, f), m(r, f), N(r, f), \bar{N}(r, f), \ldots$ (see [6], [8]). We denote by $S(r, f)$ any function satisfying $S(r, f)=o\{T(r, f)\}$, as $r \rightarrow+\infty$, possibly outside of finite measure. Let $a$ be an arbitrary complex number and $k$ be a positive integer. We denote by $N_{k)}\left(r, \frac{1}{f-a}\right)$ or $N_{k)}(r, a, f)$ the counting function for the zeros of $f(z)-a$ with multiplicity $\leq k$ and by $\bar{N}_{k)}(r, a, f)$ the counting function for the zeros of $f(z)-a$ with multiplicity $\leq k$, counting only once. We use $\bar{E}_{k)}(a, f)=\{z /$ zeros of $f(z)-a$ with multiplicity $\leq k$, where each zero being counted only once\}. Further we denote by $N_{2}\left(r, \frac{1}{f-a}\right)$ the counting function for the zeros of $f(z)-a$ where a simple zero is counted once and a mutiple zero is counted twice. Similarly, we can define $N_{2}(r, f)$. We denote by $\bar{N}_{2}\left(r, \frac{1}{f-a}\right)$ the counting function for the zeros of $f(z)-a$ where a simple zero and multiple zero is counted only once. Similarly, we can define $\bar{N}_{2}(r, f)$.

We say $f$ and $g$ share the value $a$ CM if $f(z)-a$ and $g(z)-a$ have the same zeros with the same multiplicities. We define

$$
\Theta_{2}(a, f)=1-\limsup _{r \rightarrow \infty} \frac{\bar{N}_{2}(r, a, f)}{T(r, f)}, \delta_{2}(a, f)=1-\limsup _{r \rightarrow \infty} \frac{N_{2}(r, a, f)}{T(r, f)}
$$

Corresponding author: Subhas S. Bhoosnurmath. 2000 Mathematics Subject Classification. 30D35.

Key words and phrases. Nevanlinna theory, Uniqueness, meromorphic functions ,differential polynomial and shared value.

First author was supported by the DST grant of India. Project No. SR/S4/MS:520/08. 
Definition 1. Any expression of the type

$$
P(f)=\sum_{i=1}^{n} \alpha_{i}(z) f^{n_{i_{0}}}\left(f^{\prime}\right)^{n_{i_{1}}}\left(f^{\prime \prime}\right)^{n_{i_{2}}} \cdots\left(f^{(m)}\right)^{n_{i_{m}}},
$$

is called a differential polynomial in $f$ of degree $\bar{d}(P)$, lower degree $\underline{d}(P)$ and weight $\Gamma_{P}$ where for each $i=1,2 \ldots n, n_{i_{0}}, n_{i_{1}} \ldots, n_{i_{m}}$ are non-negative integers, $\alpha_{i}=\alpha_{i}(z)$ are meromorphic functions satisfying $T\left(r, \alpha_{i}\right)=S(r, f)$ and

$$
\bar{d}(P)=\max \left\{\sum_{j=0}^{m} n_{i_{j}}: 1 \leq i \leq n\right\}, \quad \underline{d}(P)=\min \left\{\sum_{j=0}^{m} n_{i_{j}}: 1 \leq i \leq n\right\}
$$

and

$$
\Gamma_{P}=\max \left\{\sum_{j=0}^{m}(j+1) n_{i_{j}}: 1 \leq i \leq n\right\} .
$$

In 1989, H. X. Yi [5] proved the following theorem .

Theorem A. Let $f_{1}(z)$ and $f_{2}(z)$ be non-constant meromorphic functions, $b_{j}(j=1,2,3)$ be three distinct finite non-zero complex numbers, $k$ be a positive integer or $\infty$, and $n$ be a positive integer satisfying

$$
\bar{E}_{k)}\left(b_{j}, f_{1}^{(n)}\right)=\bar{E}_{k)}\left(b_{j}, f_{2}^{(n)}\right)
$$

Furthermore, let

$C_{i}=3(k+1) \delta\left(0, f_{i}\right)+(2 n k+3 n+k+1) \Theta\left(\infty, f_{i}\right)-(2 n k+3 n+3 k+4)(i=1,2)$.

If

$$
\begin{aligned}
& \min \left\{C_{1}, C_{2}\right\} \geq 0, \\
& \max \left\{C_{1}, C_{2}\right\}>0
\end{aligned}
$$

then $f_{1}(z) \equiv f_{2}(z)$.

In 2007, Anupama J. Patil [1] proved the following theorem which generalises the above result to differential polynomials in $f$ and also improve the conditions in the above theorem.

Theorem B. Let $f_{1}(z)$ and $f_{2}(z)$ be two non-constant meromorphic functions and $P\left(f_{1}\right)$ and $P\left(f_{2}\right)$ be non-constant differential polynomials in $f_{1}$ and $f_{2}$ respectively. Let $\alpha_{j}(\not \equiv 0, \infty)(j=$ $1,2,3)$ be three non-zero distinct entire small functions of $P\left(f_{1}\right)$ and $P\left(f_{2}\right), k_{1} \geq k_{2} \geq k_{3}$ be positive integers or $\infty$ and $n$ be a positive integer satisfying

$$
\bar{E}_{\left.k_{i}\right)}\left(\alpha_{j}, P\left(f_{1}\right)\right)=\bar{E}_{\left.k_{i}\right)}\left(\alpha_{j}, P\left(f_{2}\right)\right), \quad i, j=1,2,3 .
$$

Furthermore, let

$D_{i}=3\left(k_{1}+1\right)\left(k_{3}+1\right) \delta_{m+1}\left(a, f_{i}\right)-\left(\bar{d}+Q c_{i}\right)\left[6\left(k_{1}+1\right)+4 k_{1}\left(k_{3}+1\right)\right] \quad(i=1,2)$, 
where $\bar{d}=\bar{d}\left(P\left(f_{1}\right)(z)\right)=\bar{d}\left(P\left(f_{2}\right)(z)\right)=\max \left\{\sum_{j=0}^{m} n_{i_{j}} ; 1 \leq i \leq n\right\}$, $Q=\max \left\{n_{i_{1}}+2 n_{i_{2}}+3 n_{i_{3}}+\cdots \cdots+m n_{i_{m}} ; 1 \leq i \leq n\right\}, m=$ order of the highest derivative of $f$ occurring in $P$ and $c_{i}=1-\Theta\left(\infty, f_{i}\right)$. If

$$
\begin{aligned}
& \min \left(D_{1}, D_{2}\right) \geq 0 \\
& \max \left(D_{1}, D_{2}\right)>0
\end{aligned}
$$

then $P\left(f_{1}\right)(z) \equiv P\left(f_{2}\right)(z)$.

As a corollary to the above theorem, Anupama J. Patil [1] improve Theorem A by considering three non-zero small entire functions of $f^{(k)}$ instead of three non-zero complex numbers.

Corollary B. Let $f_{1}(z)$ and $f_{2}(z)$ be non-constant meromorphic functions, $b_{j}(j=1,2,3)$ be three non-zero entire small functions of $f_{1}^{(m)}$ and $f_{2}^{(m)}, k$ be a positive integer or $\infty$, and $m$ be a positive integer satisfying

$$
\bar{E}_{k)}\left(b_{j}, f_{1}^{(m)}\right)=\bar{E}_{k)}\left(b_{j}, f_{2}^{(m)}\right)
$$

Furthermore, let $C_{i}=3(k+1) \delta\left(0, f_{i}\right)+(2 m k+3 m+k+1) \Theta\left(\infty, f_{i}\right)-(2 m k+3 m+3 k+4)(i=1,2)$. If

$$
\begin{aligned}
& \min \left\{C_{1}, C_{2}\right\} \geq 0 \\
& \max \left\{C_{1}, C_{2}\right\}>0
\end{aligned}
$$

then $f_{1}(z) \equiv f_{2}(z)$.

In this paper,we study the uniqueness problems on meromorphic functions concerning differential polynomials that share three entire small functions as an application of Theorem B. Here our techniques employed are much different and relatively simple and lead to several significant results. They also throw new light on such topics.

\section{MAIN RESULTS}

Theorem 1. Let $f$ and $g$ be two non-constant meromorphic functions and $\alpha_{j}(\not \equiv 0, \infty)(j=$ $1,2,3)$ be three non-zero distinct entire small functions, $k$ be a positive integer or $\infty$ satisfying

$$
\bar{E}_{k)}\left(\alpha_{j}, f^{n}\left(f^{p}-1\right) f^{\prime}\right)=\bar{E}_{k)}\left(\alpha_{j}, g^{n}\left(g^{p}-1\right) g^{\prime}\right), \quad j=1,2,3,
$$

where $n$ and $p$ are positive integers, then either $f \equiv g$ or

$$
g=\left[\frac{(n+p+1)\left(h^{n+1}-1\right)}{(n+1)\left(h^{n+p+1}-1\right)}\right]^{1 / p}, \quad f=\left[\frac{(n+p+1) h^{p}\left(h^{n+1}-1\right)}{(n+1)\left(h^{n+p+1}-1\right)}\right]^{1 / p}
$$

where $h$ is a non-constant meromorphic function. 
Letting $k \rightarrow \infty$ and $p=1$ in the above theorem, we have the following result.

Corollary 1.1. Let $f$ and $g$ be two non-constant meromorphic functions and $\alpha_{j}(\equiv \equiv, \infty)(j=$ $1,2,3)$ be three non-zero distinct entire small functions satisfying

$$
\bar{E}\left(\alpha_{j}, f^{n}(f-1) f^{\prime}\right)=\bar{E}\left(\alpha_{j}, g^{n}(g-1) g^{\prime}\right), \quad j=1,2,3,
$$

where $n$ is a positive integer, then either $f \equiv g$ or $g=\left[(n+2)\left(h^{n+1}-1\right)\right] /\left[(n+1)\left(h^{n+2}-1\right)\right]$, $f=\left[(n+2) h\left(h^{n+1}-1\right)\right] /\left[(n+1)\left(h^{n+2}-1\right)\right]$, where $h$ is a non-constant meromorphic function.

Theorem 2. Let $f$ and $g$ be two non-constant meromorphic functions and $\alpha_{j}(\not \equiv 0, \infty)(j=$ $1,2,3)$ be three non-zero distinct entire small functions, $k$ be a positive integer or $\infty$ and $p$ is a positive integer satisfying

$$
\bar{E}_{k)}\left(\alpha_{j}, f^{n}(f-1)^{p} f^{\prime}\right)=\bar{E}_{k)}\left(\alpha_{j}, g^{n}(g-1)^{p} g^{\prime}\right), \quad j=1,2,3,
$$

(i) if $p=1$ and $n$ is a positive integer, then either $f \equiv g$ or $g=\left[(n+2)\left(h^{n+1}-1\right)\right] /\left[(n+1)\left(h^{n+2}-\right.\right.$ $1)], f=\left[(n+2) h\left(h^{n+1}-1\right)\right] /\left[(n+1)\left(h^{n+2}-1\right)\right]$, where $h$ is a non-constant meromorphic function.

(ii) if $p=2$ and $n \geq 3$, then $f \equiv g$.

(iii) if $p>2$ and $n$ is a positive integer, then

$$
f^{n+1} \sum_{l=0}^{p} \frac{(-1)^{l} C_{p}^{l}}{n+p-l+1} f^{p-l} \equiv g^{n+1} \sum_{l=0}^{p} \frac{(-1)^{l} C_{p}^{l}}{n+p-l+1} g^{p-l} .
$$

Theorem 3. Let $f$ and $g$ be two non-constant meromorphic functions and $\alpha_{j}(\not \equiv 0, \infty)(j=$ $1,2,3)$ be three non-zero distinct entire small functions, $k$ be a positive integer or $\infty$ satisfying

$$
\bar{E}_{k)}\left(\alpha_{j}, f^{n} f^{\prime}\right)=\bar{E}_{k)}\left(\alpha_{j}, g^{n} g^{\prime}\right), \quad j=1,2,3
$$

where $n$ is positive integer, then either $f \equiv g$ or $h^{n+1}-1=0$, where $h$ is a non-constant meromorphic function.

Letting $k \rightarrow \infty$, we have the following result.

Corollary 3.1. Let $f$ and $g$ be two non-constant meromorphic functions and $\alpha_{j}(\equiv 0, \infty)(j=$ $1,2,3)$ be three non-zero distinct entire small functions satisfying

$$
\bar{E}\left(\alpha_{j}, f^{n} f^{\prime}\right)=\bar{E}\left(\alpha_{j}, g^{n} g^{\prime}\right), \quad j=1,2,3
$$

where $n$ is a positive integer, then either $f \equiv g$ or $h^{n+1}-1=0$, where $h$ is a non-constant meromorphic function. 


\section{Lemmas}

In order to prove our results, we need the following lemmas.

Lemma 2.1.([2]) Let $f(z)$ be a non-constant meromorphic function and let

$$
R(f)=\sum_{k=0}^{n} a_{k} f^{k} / \sum_{j=0}^{m} b_{j} f^{j}
$$

be an irreducible rational function in $f$ with constant coefficients $\left\{a_{k}\right\}$ and $\left\{b_{j}\right\}$, where $a_{n} \neq 0$ and $b_{m} \neq 0$. Then

$$
T(r, R(f))=d T(r, f)+S(r, f),
$$

where $d=\max \{n, m\}$.

Lemma 2.2.([6]) Suppose that $f(z)$ is a non-constant meromorphic function in the complex plane and $a(\in \mathbb{C} \cup\{\infty\})$ is any complex number. Then

$$
\sum_{a} \Theta(a, f) \leq 2
$$

Lemma 2.3.([4]) Let

$$
Q(\omega)=(n-1)^{2}\left(\omega^{n}-1\right)\left(\omega^{n-2}-1\right)-n(n-2)\left(\omega^{n-1}-1\right)^{2},
$$

then

$$
Q(\omega)=(\omega-1)^{4}\left(\omega-\beta_{1}\right)\left(\omega-\beta_{2}\right) \ldots\left(\omega-\beta_{2 n-6}\right) .
$$

where $\beta_{j} \in C-[0,1](j=1,2, \ldots, 2 n-6)$, which are distinct respectively.

Lemma 2.4. ([3]) Let $P(z)=a_{n} z^{n}+a_{n-1} z^{n-1}+\ldots+a_{0}$, where $a_{n}(\neq 0), a_{n-1}, \ldots, a_{0}$ are constants. If $f(z)$ is a meromorphic function, then

$$
T(r, P(f))=n T(r, f)+S(r, f) .
$$

Lemma 2.5.([7]) Let $f$ be a meromorphic function and $P(f)$ be a differential polynomial in $f$. Then

$$
\begin{gathered}
T(r, P(f))=Q \bar{N}(r, f)+\bar{d}(P) T(r, f)+S(r, f) \\
\bar{d}(P)=\max \left\{\sum_{j=0}^{m} n_{i_{j}} ; 1 \leq i \leq n\right\}, Q=\max \left\{n_{i_{1}}+2 n_{i_{2}}+3 n_{i_{3}}+\cdots \cdots+m n_{i_{m}} ; 1 \leq i \leq n\right\} .
\end{gathered}
$$

Theorem 2.1.([7]) Let $f$ be a meromorphic function and let $P(f)$ be a non-constant differential polynomial in $f$ such that every term in $P(f)$ contains at least one of the derivatives of $f$. Let $m$ 
be the order of the highest derivative of $f$ occuring in $P(f)$. If $k$ is a positive integer $\leq m$ such that every term in $P(f)$ contains atleast one of $f^{\prime}, f^{\prime \prime}, \ldots, f^{(k)}$, then

$$
d_{k} T(r, f) \leq d_{k} N_{k+1}(r, a, f)+\bar{N}\left(r, \frac{1}{P(f)-b}\right)+\bar{N}\left(r, \frac{1}{P(f)-c}\right)+S(r, f)
$$

where $a \in C, b(\equiv 0), c(\not \equiv 0)$ are distinct meromorphic functions satisfying $T(r, b)=S(r, P)$, $T(r, c)=S(r, P)$ and $d_{k}=\min \left\{\sum_{j=1}^{k} n_{i_{j}}: 1 \leq i \leq n\right\}$.

\section{Proof of Theorems}

The proof of Theorem B is in [7]. However, we give the proof for the sake of completeness.

Proof of Theorem B. Let

$$
\begin{aligned}
& P\left(f_{1}\right)(z)=P_{1}(z) \\
& P\left(f_{2}\right)(z)=P_{2}(z) .
\end{aligned}
$$

From the Theorem 2.1, we have for the meromorphic function $f_{1}$, its differential polynomial $P_{1}$, any element $a \in C$ and two distinct non-zero small functions $\alpha, \beta$ of $P\left(f_{1}\right)$,

$$
d_{k} T\left(r, f_{1}\right) \leq d_{k} N_{m+1}\left(r, a, f_{1}\right)+\bar{N}\left(r, \frac{1}{P_{1}-\alpha}\right)+\bar{N}\left(r, \frac{1}{P_{1}-\beta}\right)+S\left(r, f_{1}\right)
$$

Since $d_{k} \geq 1$,

$$
T\left(r, f_{1}\right) \leq N_{m+1}\left(r, a, f_{1}\right)+\bar{N}\left(r, \frac{1}{P_{1}-\alpha}\right)+\bar{N}\left(r, \frac{1}{P_{1}-\beta}\right)+S\left(r, f_{1}\right)
$$

where $m$ is the order of the highest derivative of $f$ occuring in $P_{1}$. Since there are $C_{2}^{3}$ ways of selecting one element $a$ and two elements from $\alpha_{1}, \alpha_{2}, \alpha_{3}$. For a given $a \in C$ and three non-zero distinct entire small functions $\alpha_{1}, \alpha_{2}, \alpha_{3}$ of $P_{1}$ and $P_{2}$,we have

$$
\begin{aligned}
& T\left(r, f_{1}\right) \leq N_{m+1}\left(r, a, f_{1}\right)+\bar{N}\left(r, \frac{1}{P_{1}-\alpha_{1}}\right)+\bar{N}\left(r, \frac{1}{P_{1}-\alpha_{2}}\right)+S\left(r, f_{1}\right) \\
& T\left(r, f_{1}\right) \leq N_{m+1}\left(r, a, f_{1}\right)+\bar{N}\left(r, \frac{1}{P_{1}-\alpha_{2}}\right)+\bar{N}\left(r, \frac{1}{P_{1}-\alpha_{3}}\right)+S\left(r, f_{1}\right) \\
& T\left(r, f_{1}\right) \leq N_{m+1}\left(r, a, f_{1}\right)+\bar{N}\left(r, \frac{1}{P_{1}-\alpha_{1}}\right)+\bar{N}\left(r, \frac{1}{P_{1}-\alpha_{3}}\right)+S\left(r, f_{1}\right)
\end{aligned}
$$

adding all the above equations, we get

$$
\begin{aligned}
3 T\left(r, f_{1}\right) & \leq 3 N_{m+1}\left(r, a, f_{1}\right)+2 \sum_{i=1}^{3} \bar{N}\left(r, \frac{1}{P_{1}-\alpha_{i}}\right)+S\left(r, f_{1}\right) \\
& \leq 3\left(1-\delta_{m+1}\left(a, f_{1}\right)\right) T\left(r, f_{1}\right)+2 \sum_{i=1}^{3} \bar{N}\left(r, \frac{1}{P_{1}-\alpha_{i}}\right)+S\left(r, f_{1}\right)
\end{aligned}
$$


i.e.,

$$
\begin{aligned}
3 \delta_{m+1}\left(a, f_{1}\right) T\left(r, f_{1}\right) & \leq 2 \sum_{i=1}^{3} \bar{N}\left(r, \frac{1}{P_{1}-\alpha_{i}}\right)+S\left(r, f_{1}\right) \\
& \leq 2 \sum_{i=1}^{3}\left[\frac{k_{i}}{k_{i}+1} \bar{N}_{\left.k_{i}\right)}\left(r, \frac{1}{P_{1}-\alpha_{i}}\right)+\frac{1}{k_{i}+1} N\left(r, \frac{1}{P_{1}-\alpha_{i}}\right)\right]+S\left(r, f_{1}\right) \\
& \leq 2 \sum_{i=1}^{3} \frac{k_{i}}{k_{i}+1} \bar{N}_{\left.k_{i}\right)}\left(r, \frac{1}{P_{1}-\alpha_{i}}\right)+2 \sum_{i=1}^{3} \frac{1}{k_{i}+1} N\left(r, \frac{1}{P_{1}-\alpha_{i}}\right)+S\left(r, f_{1}\right) \\
& \leq 2 \frac{k_{1}}{k_{1}+1} \sum_{i=1}^{3} \bar{N}_{\left.k_{i}\right)}\left(r, \frac{1}{P_{1}-\alpha_{i}}\right)+\frac{6}{k_{3}+1} T\left(r, P_{1}\right)+S\left(r, f_{1}\right) .
\end{aligned}
$$

By Lemma 2.5, we have

$$
\begin{aligned}
T\left(r, P_{1}\right) & =Q \bar{N}\left(r, f_{1}\right)+\bar{d}\left(P_{1}\right) T\left(r, f_{1}\right)+S\left(r, f_{1}\right) \\
& =Q\left(1-\Theta\left(\infty, f_{1}\right)\right) T\left(r, f_{1}\right)+\bar{d}\left(P_{1}\right) T\left(r, f_{1}\right)+S\left(r, f_{1}\right) .
\end{aligned}
$$

Therefore

$$
\begin{aligned}
3 \delta_{m+1}\left(a, f_{1}\right) T\left(r, f_{1}\right) & \leq 2 \frac{k_{1}}{k_{1}+1} \sum_{i=1}^{3} \bar{N}_{\left.k_{i}\right)}\left(r, \frac{1}{P_{1}-\alpha_{i}}\right)+\frac{6\left(\bar{d}+Q\left(1-\Theta\left(\infty, f_{1}\right)\right)\right)}{k_{3}+1} T\left(r, f_{1}\right)+S\left(r, f_{1}\right) \\
\text { i.e., } \quad d_{1} T\left(r, f_{1}\right) & \leq 2 k_{1}\left(k_{3}+1\right) \sum_{i=1}^{3} \bar{N}_{\left.k_{i}\right)}\left(r, \frac{1}{P_{1}-\alpha_{i}}\right)+S\left(r, f_{1}\right)
\end{aligned}
$$

where

$$
d_{i}=3 \delta_{m+1}\left(a, f_{i}\right)\left(k_{1}+1\right)\left(k_{3}+1\right)-6\left(\bar{d}+Q\left(1-\Theta\left(\infty, f_{i}\right)\right)\right)\left(k_{1}+1\right), \quad(i=1,2) .
$$

Similarly, we have

$$
d_{2} T\left(r, f_{2}\right) \leq 2 k_{1}\left(k_{3}+1\right) \sum_{i=1}^{3} \bar{N}_{\left.k_{i}\right)}\left(r, \frac{1}{P_{2}-\alpha_{i}}\right)+S\left(r, f_{2}\right)
$$

Adding the above two equations, we get

$$
\left.d_{1} T\left(r, f_{1}\right)+d_{2} T\left(r, f_{2}\right) \leq 2 k_{1}\left(k_{3}+1\right) \sum_{i=1}^{3}\left[\bar{N}_{\left.k_{i}\right)}\left(r, \frac{1}{P_{1}-\alpha_{i}}\right)+\bar{N}_{k_{i}}\right)\left(r, \frac{1}{P_{2}-\alpha_{i}}\right)\right]+S\left(r, f_{1}\right)+S\left(r, f_{2}\right)
$$

Since by hypothesis,

$$
\bar{E}_{\left.k_{i}\right)}\left(\alpha_{j}, P\left(f_{1}\right)\right)=\bar{E}_{\left.k_{i}\right)}\left(\alpha_{j}, P\left(f_{2}\right)\right) \quad(i, j=1,2,3)
$$

so that

$$
\bar{N}_{\left.k_{i}\right)}\left(r, \frac{1}{P_{1}-\alpha_{i}}\right)=\bar{N}_{\left.k_{i}\right)}\left(r, \frac{1}{P_{2}-\alpha_{i}}\right)=N_{0}^{\left.k_{i}\right)}\left(r, \alpha_{i}\right) .
$$


Thus

$$
\begin{aligned}
& \left.\left.d_{1} T\left(r, f_{1}\right)+d_{2} T\left(r, f_{2}\right) \leq 2 k_{1}\left(k_{3}+1\right) \sum_{i=1}^{3}\left[\bar{N}_{k_{i}}\right)\left(r, \frac{1}{P_{1}-\alpha_{i}}\right)+\bar{N}_{k_{i}}\right)\left(r, \frac{1}{P_{2}-\alpha_{i}}\right)\right]+S\left(r, f_{1}\right)+S\left(r, f_{2}\right) \\
& d_{1} T\left(r, f_{1}\right)+d_{2} T\left(r, f_{2}\right) \leq 4 k_{1}\left(k_{3}+1\right) \sum_{i=1}^{3} N_{0}^{\left.k_{i}\right)}\left(r, \alpha_{i}\right)+S\left(r, f_{1}\right)+S\left(r, f_{2}\right) .
\end{aligned}
$$

Suppose,

$$
P_{1}(z) \not \equiv P_{2}(z)
$$

Then under the assumption that $P_{1}$ and $P_{2}$ are distinct, it follows that for any $\alpha \in S\left(P_{1}\right) \cap S\left(P_{2}\right)-$ $\{0, \infty\}$, each common zero of $P_{1}-\alpha$ and $P_{2}-\alpha$ is a zero of $P_{1}-P_{2}$. Since $\alpha_{1}, \alpha_{2}, \alpha_{3}$ are distinct, we have

$$
\begin{aligned}
\sum_{i=1}^{3} N_{0}^{\left.k_{i}\right)}\left(r, \alpha_{i}\right) \leq & N\left(r, \frac{1}{P_{1}-P_{2}}\right) \leq T\left(r, P_{1}-P_{2}\right) \\
\leq & T\left(r, P_{1}\right)+T\left(r, P_{2}\right)+O(1) \\
\sum_{i=1}^{3} N_{0}^{\left.k_{i}\right)}\left(r, \alpha_{i}\right) \leq & \left(\bar{d}+Q\left(1-\Theta\left(\infty, f_{1}\right)\right)\right) T\left(r, f_{1}\right)+S\left(r, f_{1}\right) \\
& +\left(\bar{d}+Q\left(1-\Theta\left(\infty, f_{2}\right)\right)\right) T\left(r, f_{2}\right)+S\left(r, f_{2}\right)
\end{aligned}
$$

From equations (3) and (5), we get

$$
D_{1} T\left(r, f_{1}\right)+D_{2} T\left(r, f_{2}\right) \leq S\left(r, f_{1}\right)+S\left(r, f_{2}\right)
$$

where for $i=1,2, D_{i}=d_{i}-4 k_{1}\left(k_{3}+1\right)\left(\bar{d}+Q\left(1-\Theta\left(\infty, f_{i}\right)\right)\right)$ which is required $D_{i}$ mentioned earlier. From conditions (1), (2) the above inequility (6) is not possible. Thus our assumption (4) is not true and hence we must have

$$
\begin{aligned}
P_{1}(z) & \equiv P_{2}(z) \\
\text { i.e., } P\left(f_{1}\right)(z) & \equiv P\left(f_{2}\right)(z) .
\end{aligned}
$$

Proof of Theorem 1. First, we need to show that $f^{n}\left(f^{p}-1\right) f^{\prime} \equiv g^{n}\left(g^{p}-1\right) g^{\prime}$. Consider $k_{1}=$ $k_{2}=k_{3}=k$ and $a=0$. We have $\bar{d}\left\{f^{n}\left(f^{p}-1\right) f^{\prime}\right\}=\bar{d}\left\{g^{n}\left(g^{p}-1\right) g^{\prime}\right\}=n+p+1, Q=1$ and $m=1$. Therefore

$$
\min \left\{D_{f}, D_{g}\right\} \geq 0, \quad \max \left\{D_{f}, D_{g}\right\}>0
$$

where

$$
D_{f}=3(k+1)^{2} \delta_{2}(0, f)-(n+p+2)(k+1)(6+4 k)+(k+1)(6+4 k) \Theta(\infty, f)
$$

and 


$$
D_{g}=3(k+1)^{2} \delta_{2}(0, g)-(n+p+2)(k+1)(6+4 k)+(k+1)(6+4 k) \Theta(\infty, g) .
$$

By Theorem B, we get

$$
\begin{aligned}
f^{n}\left(f^{p}-1\right) f^{\prime} & \equiv g^{n}\left(g^{p}-1\right) g^{\prime} \\
\left(F^{*}\right)^{\prime} & \equiv\left(G^{*}\right)^{\prime}
\end{aligned}
$$

Then

$$
F^{*} \equiv G^{*}+c, \quad c \text { is a constant }
$$

where

$$
F^{*}=\frac{f^{n+p+1}}{n+p+1}-\frac{f^{n+1}}{n+1}, \quad G^{*}=\frac{g^{n+p+1}}{n+p+1}-\frac{g^{n+1}}{n+1} .
$$

By Lemma 2.1, we have $T\left(r, F^{*}\right)=(n+p+1) T(r, f)+S(r, f)$. Note that

$$
\begin{aligned}
\bar{N}_{2}\left(r, \frac{1}{F^{*}}\right) & =\bar{N}_{2}\left(r, \frac{1}{f}\right)+\overline{N_{2}}\left(r, \frac{1}{\frac{f^{p}}{n+p+1}-\frac{1}{n+1}}\right) \\
& \leq \bar{N}_{2}\left(r, \frac{1}{f}\right)+T\left(r, \frac{1}{\frac{f^{p}}{n+p+1}-\frac{1}{n+1}}\right) \\
& =\bar{N}_{2}\left(r, \frac{1}{f}\right)+p T(r, f)+S(r, f) .
\end{aligned}
$$

So,

$$
\frac{\bar{N}_{2}\left(r, \frac{1}{F^{*}}\right)}{T\left(r, F^{*}\right)} \leq \frac{\bar{N}_{2}\left(r, \frac{1}{f}\right)}{(n+p+1) T(r, f)+S(r, f)}+\frac{p T(r, f)}{(n+p+1) T(r, f)+S(r, f)}
$$

Therefore,

$$
\begin{aligned}
\limsup _{r \rightarrow \infty} \frac{\bar{N}_{2}\left(r, \frac{1}{F^{*}}\right)}{T\left(r, F^{*}\right)} & \leq \frac{\limsup _{r \rightarrow \infty} \overline{N_{2}}\left(r, \frac{1}{f}\right) / T(r, f)}{n+p+1}+\frac{p}{n+p+1} \\
1-\Theta_{2}\left(0, F^{*}\right) & \leq \frac{1-\Theta_{2}(0, f)}{n+p+1}+\frac{p}{n+p+1} \\
\text { i.e, } \quad \Theta_{2}\left(0, F^{*}\right) & \geq \frac{n}{n+p+1}+\frac{\Theta_{2}(0, f)}{n+p+1}
\end{aligned}
$$

Similarly, we have

$$
\Theta_{2}\left(0, G^{*}\right) \geq \frac{n}{n+p+1}+\frac{\Theta_{2}(0, g)}{n+p+1}
$$

Note that $\bar{N}_{2}\left(r, F^{*}\right)=\bar{N}_{2}(r, f)$. So,

$$
\frac{\bar{N}_{2}\left(r, F^{*}\right)}{T\left(r, F^{*}\right)}=\frac{\bar{N}_{2}(r, f)}{(n+p+1) T(r, f)+S(r, f)}
$$




$$
\begin{aligned}
\limsup _{r \rightarrow \infty} \frac{\bar{N}_{2}\left(r, F^{*}\right)}{T\left(r, F^{*}\right)} & =\frac{\limsup _{r \rightarrow \infty} \frac{\bar{N}_{2}(r, f)}{T(r, f)}}{(n+p+1)} \\
1-\Theta_{2}\left(\infty, F^{*}\right) & =\frac{1-\Theta_{2}(\infty, f)}{n+p+1} \\
\Theta_{2}\left(\infty, F^{*}\right) & =\frac{n+p}{n+p+1}+\frac{\Theta_{2}(\infty, f)}{n+p+1}
\end{aligned}
$$

And, by the definition we have,

$$
\begin{aligned}
\Theta_{2}\left(c, F^{*}\right) & =1-\limsup _{r \rightarrow \infty} \frac{\bar{N}_{2}\left(r, \frac{1}{F^{*}-c}\right)}{T\left(r, F^{*}\right)} \\
& =1-\limsup _{r \rightarrow \infty} \frac{\bar{N}_{2}\left(r, \frac{1}{G^{*}}\right)}{T\left(r, F^{*}\right)}, \quad \text { by (7) }
\end{aligned}
$$

Since $F^{*} \equiv G^{*}+c, c$ a constant, so $T\left(r, F^{*}\right)=T\left(r, G^{*}\right)$. Therefore,

$$
\Theta_{2}\left(c, F^{*}\right)=1-\limsup _{r \rightarrow \infty} \frac{\bar{N}_{2}\left(r, \frac{1}{G^{*}}\right)}{T\left(r, G^{*}\right)}=\Theta_{2}\left(0, G^{*}\right)
$$

We now show that $c=0$ in (7). Suppose that $c \neq 0$, then

$$
\begin{aligned}
& \Theta_{2}\left(0, F^{*}\right)+\Theta_{2}\left(\infty, F^{*}\right)+\Theta_{2}\left(c, F^{*}\right) \\
& \geq \frac{n}{n+p+1}+\frac{\Theta_{2}(0, f)}{n+p+1}+\frac{n+p}{n+p+1}+\frac{\Theta_{2}(\infty, f)}{n+p+1}+\frac{n}{n+p+1}+\frac{\Theta_{2}(0, g)}{n+p+1} \\
& \geq \frac{n}{n+p+1}+\frac{\delta_{2}(0, f)}{n+p+1}+\frac{n+p}{n+p+1}+\frac{\Theta(\infty, f)}{n+p+1}+\frac{n}{n+p+1}+\frac{\delta_{2}(0, g)}{n+p+1}
\end{aligned}
$$

because $\bar{N}_{2}(r, f)=\bar{N}(r, f)$ and hence

$$
\limsup _{r \rightarrow \infty} \frac{\bar{N}_{2}(r, f)}{T(r, f)}=\limsup _{r \rightarrow \infty} \frac{\bar{N}(r, f)}{T(r, f)} \Rightarrow \Theta_{2}(\infty, f)=\Theta(\infty, f) .
$$

Since $\min \left\{D_{f}, D_{g}\right\} \geq 0$,

$$
3(k+1)^{2} \delta_{2}(0, f)-(n+p+2)(k+1)(6+4 k)+(k+1)(6+4 k) \Theta(\infty, f) \geq 0
$$

and

$$
3(k+1)^{2} \delta_{2}(0, g)-(n+p+2)(k+1)(6+4 k)+(k+1)(6+4 k) \Theta(\infty, g) \geq 0 \text {. }
$$

Therefore

$$
\begin{aligned}
& \delta_{2}(0, f) \geq \frac{(n+p+2)(6+4 k)}{3(k+1)}-\frac{(6+4 k)}{3(k+1)} \Theta(\infty, f) \\
& \delta_{2}(0, g) \geq \frac{(n+p+2)(6+4 k)}{3(k+1)}-\frac{(6+4 k)}{3(k+1)} \Theta(\infty, g)
\end{aligned}
$$


Substituting (9) and (10) in (8), we get

$$
\begin{aligned}
& \Theta_{2}\left(0, F^{*}\right)+\Theta_{2}\left(\infty, F^{*}\right)+\Theta_{2}\left(c, F^{*}\right) \\
& \geq \frac{3 n+p}{n+p+1}+\frac{1}{n+p+1}\left\{\frac{(n+p+2)(6+4 k)}{3(k+1)}-\frac{(6+4 k)}{3(k+1)} \Theta(\infty, f)\right\}+\frac{\Theta(\infty, f)}{n+p+1} \\
& \quad+\frac{1}{n+p+1}\left\{\frac{(n+p+2)(6+4 k)}{3(k+1)}-\frac{(6+4 k)}{3(k+1)} \Theta(\infty, g)\right\} \\
& =\frac{3 n+p}{n+p+1}+\frac{2(n+p+2)(6+4 k)}{3(k+1)(n+p+1)}-\frac{(k+3)}{3(k+1)(n+p+1)} \Theta(\infty, f)-\frac{(6+4 k)}{3(k+1)(n+p+1)} \Theta(\infty, g) \\
& \geq \frac{3 n+p}{n+p+1}+\frac{2(n+p+2)(6+4 k)}{3(k+1)(n+p+1)}-\frac{(k+3)}{3(k+1)(n+p+1)}-\frac{(6+4 k)}{3(k+1)(n+p+1)} \\
& =\frac{17 k n+11 k p+21 n+15 p+11 k+15}{3(k+1)(n+p+1)}>2 .
\end{aligned}
$$

Because, let

$$
H_{n}=\frac{17 k n+11 k p+21 n+15 p+11 k+15}{3(k+1)(n+p+1)}, \quad k>0, \quad p>0 .
$$

Then

$$
H_{n}^{\prime}=\frac{6 k p+6 p+6 k+6}{3(k+1)(n+p+1)^{2}}>0 \quad \text { for } \quad k>0, \quad p>0 .
$$

Thus $H_{n}$ is an increasing function and

$$
H_{n} \quad \text { at } \quad\{p=1, n=1\}=\frac{26 k+62}{9 k+9} \quad \text { and } \quad \lim _{k \rightarrow \infty}\left\{\frac{26 k+62}{9 k+9}\right\}=\frac{26}{9}=2.888 \ldots
$$

This shows that $H_{n}$ always exceeds the value 2, which contradicts Lemma 2.2. Hence $c=0$. Therefore

$$
\begin{aligned}
F^{*} & \equiv G^{*} \\
\text { i.e, } \frac{f^{n+p+1}}{n+p+1}-\frac{f^{n+1}}{n+1} & =\frac{g^{n+p+1}}{n+p+1}-\frac{g^{n+1}}{n+1} \\
f^{n+1}\left\{\frac{f^{p}}{n+p+1}-\frac{1}{n+1}\right\} & =g^{n+1}\left\{\frac{g^{p}}{n+p+1}-\frac{1}{n+1}\right\}
\end{aligned}
$$

Now, let $h=\frac{f}{g}$. If $h \equiv 1$, then $f \equiv g$. Suppose $h \not \equiv 1$, then

$$
\begin{gathered}
\left(\frac{f}{g}\right)^{n+1}\left\{\frac{f^{p}}{n+p+1}-\frac{1}{n+1}\right\}=\frac{g^{p}}{n+p+1}-\frac{1}{n+1} \\
h^{n+1}\left\{\frac{(h g)^{p}}{n+p+1}-\frac{1}{n+1}\right\}=\frac{g^{p}}{n+p+1}-\frac{1}{n+1}
\end{gathered}
$$




$$
\begin{aligned}
& h^{n+1}\left\{\frac{(h g)^{p}(n+1)-(n+p+1)}{(n+1)(n+p+1)}\right\}=\frac{g^{p}(n+1)-(n+p+1)}{(n+1)(n+p+1)} \\
& h^{n+p+1} g^{p}(n+1)-h^{n+1}(n+p+1)=g^{p}(n+1)-(n+p+1) \\
& h^{n+p+1} g^{p}(n+1)-g^{p}(n+1)=h^{n+1}(n+p+1)-(n+p+1) \\
& g^{p}(n+1)\left[h^{n+p+1}-1\right]=(n+p+1)\left(h^{n+1}-1\right) \\
& g^{p}=\frac{(n+p+1)\left(h^{n+1}-1\right)}{(n+1)\left(h^{n+p+1}-1\right)} .
\end{aligned}
$$

Similarly

$$
f^{p}=\frac{(n+p+1) h^{p}\left(h^{n+1}-1\right)}{(n+1)\left(h^{n+p+1}-1\right)} .
$$

Therefore,

$$
f=\left[\frac{(n+p+1) h^{p}\left(h^{n+1}-1\right)}{(n+1)\left(h^{n+p+1}-1\right)}\right]^{1 / p}, \quad g=\left[\frac{(n+p+1)\left(h^{n+1}-1\right)}{(n+1)\left(h^{n+p+1}-1\right)}\right]^{1 / p},
$$

which proves the Theorem 1.

Proof of Theorem 2. First, we show that $f^{n}(f-1)^{p} f^{\prime} \equiv g^{n}(g-1)^{p} g^{\prime}$, where

$$
(f-1)^{p}=f^{p}-p f^{p-1}+\frac{p(p-1)}{2} f^{p-2}-\cdots \cdots+(-1)^{p}
$$

So,

$$
\begin{aligned}
& f^{n}(f-1)^{p} f^{\prime}=f^{n+p} f^{\prime}-p f^{n+p-1} f^{\prime}+\frac{p(p-1)}{2} f^{n+p-2} f^{\prime}-\cdots \cdots+(-1)^{p} f^{n} f^{\prime} \\
& g^{n}(g-1)^{p} g^{\prime}=g^{n+p} g^{\prime}-p g^{n+p-1} g^{\prime}+\frac{p(p-1)}{2} g^{n+p-2} g^{\prime}-\cdots \cdots+(-1)^{p} g^{n} g^{\prime}
\end{aligned}
$$

Consider $k_{1}=k_{2}=k_{3}=k$ and $a=0$. We have, $\bar{d}\left\{f^{n}(f-1)^{p} f^{\prime}\right\}=\bar{d}\left\{g^{n}(g-1)^{p} g^{\prime}\right\}=n+p+1$, $Q=1$ and $m=1$. Therefore

$$
\min \left\{D_{f}, D_{g}\right\} \geq 0 \quad, \quad \max \left\{D_{f}, D_{g}\right\}>0,
$$

where

$$
D_{f}=3(k+1)^{2} \delta_{2}(0, f)-(n+p+2)(k+1)(6+4 k)+(k+1)(6+4 k) \Theta(\infty, f)
$$

and

$$
D_{g}=3(k+1)^{2} \delta_{2}(0, g)-(n+p+2)(k+1)(6+4 k)+(k+1)(6+4 k) \Theta(\infty, g)
$$

By Theorem B, we obtain

$$
f^{n}(f-1)^{p} f^{\prime} \equiv g^{n}(g-1)^{p} g^{\prime}
$$




$$
\left(F^{*}\right)^{\prime} \equiv\left(G^{*}\right)^{\prime}
$$

Then

$$
F^{*} \equiv G^{*}+c \quad, \quad c \text { is a constant }
$$

where

$$
\begin{aligned}
& F^{*}=\frac{f^{n+p+1}}{n+p+1}-p \frac{f^{n+p}}{n+p}+\frac{p(p-1)}{2} \frac{f^{n+p-1}}{n+p-1}-\frac{p(p-1)(p-2)}{6} \frac{f^{n+p-2}}{n+p-2}+\cdots+(-1)^{p} \frac{f^{n+1}}{n+1} \\
& G^{*}=\frac{g^{n+p+1}}{n+p+1}-p \frac{g^{n+p}}{n+p}+\frac{p(p-1)}{2} \frac{g^{n+p-1}}{n+p-1}-\frac{p(p-1)(p-2)}{6} \frac{g^{n+p-2}}{n+p-2}+\cdots+(-1)^{p} \frac{g^{n+1}}{n+1}
\end{aligned}
$$

By Lemma 2.1, we have $T\left(r, F^{*}\right)=(n+p+1) T(r, f)+S(r, f)$. Note that

$$
\begin{aligned}
\bar{N}_{2}\left(r, \frac{1}{F^{*}}\right) & =\bar{N}_{2}\left(r, \frac{1}{f}\right)+\overline{N_{2}}\left(r, \frac{1}{\frac{f^{p}}{n+p+1}-p \frac{f^{p-1}}{n+p}+\frac{p(p-1)}{2} \frac{f^{p-2}}{n+p+1}-\frac{p(p-1)(p-2)}{6} \frac{f^{p-3}}{n+p-2} \ldots \ldots+\frac{(-1)^{p}}{n+1}}\right) \\
& \leq \bar{N}_{2}\left(r, \frac{1}{f}\right)+T\left(r, \frac{f^{p}}{n+p+1}-p \frac{f^{p-1}}{n+p}+\frac{p(p-1)}{2} \frac{f^{p-2}}{n+p+1} \ldots+\frac{(-1)^{p}}{n+1}\right) \\
& =\bar{N}_{2}\left(r, \frac{1}{f}\right)+p T(r, f)+S(r, f) \quad \text { (using Lemma 2.4) }
\end{aligned}
$$

So,

$$
\frac{\bar{N}_{2}\left(r, \frac{1}{F^{*}}\right)}{T\left(r, F^{*}\right)} \leq \frac{\bar{N}_{2}\left(r, \frac{1}{f}\right)}{(n+p+1) T(r, f)+S(r, f)}+\frac{p T(r, f)}{(n+p+1) T(r, f)+S(r, f)}
$$

Therefore,

i.e,

$$
\begin{aligned}
\limsup _{r \rightarrow \infty} \frac{\bar{N}_{2}\left(r, \frac{1}{F^{*}}\right)}{T\left(r, F^{*}\right)} & \leq \frac{\limsup _{r \rightarrow \infty} \overline{N_{2}}\left(r, \frac{1}{f}\right) / T(r, f)}{n+p+1}+\frac{p}{n+p+1} \\
1-\Theta_{2}\left(0, F^{*}\right) & \leq \frac{1-\Theta_{2}(0, f)}{n+p+1}+\frac{p}{n+p+1}
\end{aligned}
$$

$$
\Theta_{2}\left(0, F^{*}\right) \geq \frac{n}{n+p+1}+\frac{\Theta_{2}(0, f)}{n+p+1}
$$

Similarly, we have

$$
\Theta_{2}\left(0, G^{*}\right) \geq \frac{n}{n+p+1}+\frac{\Theta_{2}(0, g)}{n+p+1}
$$

Note that $\bar{N}_{2}\left(r, F^{*}\right)=\bar{N}_{2}(r, f)$. So,

$$
\begin{aligned}
\frac{\bar{N}_{2}\left(r, F^{*}\right)}{T\left(r, F^{*}\right)} & =\frac{\bar{N}_{2}(r, f)}{(n+p+1) T(r, f)+S(r, f)} \\
\limsup _{r \rightarrow \infty} \frac{\bar{N}_{2}\left(r, F^{*}\right)}{T\left(r, F^{*}\right)} & =\frac{\limsup _{r \rightarrow \infty} \frac{\bar{N}_{2}(r, f)}{T(r, f)}}{(n+p+1)}
\end{aligned}
$$




$$
\begin{aligned}
1-\Theta_{2}\left(\infty, F^{*}\right) & =\frac{1-\Theta_{2}(\infty, f)}{n+p+1} \\
\Theta_{2}\left(\infty, F^{*}\right) & =\frac{n+p}{n+p+1}+\frac{\Theta_{2}(\infty, f)}{n+p+1}
\end{aligned}
$$

And, by the definition we have,

$$
\begin{aligned}
\Theta_{2}\left(c, F^{*}\right) & =1-\limsup _{r \rightarrow \infty} \frac{\bar{N}_{2}\left(r, \frac{1}{F^{*}-c}\right)}{T\left(r, F^{*}\right)} \\
& =1-\limsup _{r \rightarrow \infty} \frac{\bar{N}_{2}\left(r, \frac{1}{G^{*}}\right)}{T\left(r, F^{*}\right)} .
\end{aligned}
$$

Since $F^{*} \equiv G^{*}+c, c$ a constant, so $T\left(r, F^{*}\right)=T\left(r, G^{*}\right)$. Therefore,

$$
\Theta_{2}\left(c, F^{*}\right)=1-\limsup _{r \rightarrow \infty} \frac{\bar{N}_{2}\left(r, \frac{1}{G^{*}}\right)}{T\left(r, G^{*}\right)}=\Theta_{2}\left(0, G^{*}\right)
$$

We now show that $c=0$. Proceeding as in the proof of Theorem 1 , we obtain

$$
F^{*} \equiv G^{*}
$$

(i) If $p=1$, then

$$
F^{*}=\frac{f^{n+2}}{n+2}-\frac{f^{n+1}}{n+1} \quad, \quad G^{*}=\frac{g^{n+2}}{n+2}-\frac{g^{n+1}}{n+1}
$$

Then, we can write

$$
f^{n+1}\left\{\frac{f}{n+2}-\frac{1}{n+1}\right\}=g^{n+1}\left\{\frac{g}{n+2}-\frac{1}{n+1}\right\}
$$

Now, let $h=\frac{f}{g}$. If $h \equiv 1$, then $f \equiv g$. Suppose $h \not \equiv 1$, then we easily obtain

$$
g=\frac{(n+2)\left(h^{n+1}-1\right)}{(n+1)\left(h^{n+2}-1\right)}, \quad f=\frac{(n+2) h\left(h^{n+1}-1\right)}{(n+1)\left(h^{n+2}-1\right)} .
$$

(ii) If $p=2$, then

$$
F^{*} \equiv G^{*}
$$

i.e.,

$$
\frac{f^{n+3}}{n+3}-2 \frac{f^{n+2}}{n+2}+\frac{f^{n+1}}{n+1}=\frac{g^{n+3}}{n+3}-2 \frac{g^{n+2}}{n+2}+\frac{g^{n+1}}{n+1} .
$$

Set $h=\frac{f}{g}$. Substitute $f=h g$ in (12), we obtain

$$
(n+2)(n+1) g^{2}\left(h^{n+3}-1\right)-2(n+3)(n+1) g\left(h^{n+2}-1\right)+(n+2)(n+3)\left(h^{n+1}-1\right)=0 .
$$

If $h$ is not a constant, then

$$
\left[(n+2)(n+1) g\left(h^{n+3}-1\right)-(n+3)(n+1)\left(h^{n+2}-1\right)\right]^{2}
$$




$$
\begin{aligned}
= & (n+1)^{2}(n+2)^{2} g^{2}\left(h^{n+3}-1\right)^{2}-2(n+1)(n+2)(n+3)(n+1) g\left(h^{n+3}-1\right)\left(h^{n+2}-1\right) \\
& +(n+3)^{2}(n+1)^{2}\left(h^{n+2}-1\right)^{2} \\
= & (n+2)(n+1)\left(h^{n+3}-1\right)\left[(n+2)(n+1) g^{2}\left(h^{n+3}-1\right)-2(n+3)(n+1) g\left(h^{n+2}-1\right)\right] \\
& +(n+3)^{2}(n+1)^{2}\left(h^{n+2}-1\right)^{2} \\
= & (n+2)(n+1)\left(h^{n+3}-1\right)\left\{-(n+2)(n+3)\left(h^{n+1}-1\right)\right\}+(n+3)^{2}(n+1)^{2}\left(h^{n+2}-1\right)^{2}[\text { by }(13)] \\
= & -(n+3)(n+1)\left\{(n+2)^{2}\left(h^{n+3}-1\right)\left(h^{n+1}-1\right)-(n+3)(n+1)\left(h^{n+1}-1\right)^{2}\right\}
\end{aligned}
$$

using Lemma 2.3, we get

$$
\left[(n+2)(n+1) g\left(h^{n+3}-1\right)-(n+3)(n+1)\left(h^{n+2}-1\right)\right]^{2}=-(n+3)(n+1) Q(h),
$$

where $Q(h)=(h-1)^{4}\left(h-\beta_{1}\right)\left(h-\beta_{2}\right) \ldots . .\left(h-\beta_{2 n}\right), \beta_{j} \in C-\{0,1\}(j=1,2, \ldots, 2 n)$, which are pairwise distinct.

This implies that every zero of $\left(h-\beta_{j}\right)(j=1,2, \ldots, 2 n)$ has a multiplicity of atleast 2 . By the second fundamental theorem we obtain $n \leq 2$, which is again a contradiction. Therefore, $h$ is a constant. We have from (13) that $h^{n+1}-1=0$ and $h^{n+2}-1=0$, which imply $h=1$ and hence $f \equiv g$.

(iii) If $p \geq 2$, we get

$$
\begin{aligned}
& \frac{f^{n+p+1}}{n+p+1}-p \frac{f^{n+p}}{n+p}+\frac{p(p-1)}{2} \frac{f^{n+p-1}}{n+p-1}-\frac{p(p-1)(p-2)}{6} \frac{f^{n+p-2}}{n+p-2}+\cdots+(-1)^{p} \frac{f^{n+1}}{n+1} \\
= & \frac{g^{n+p+1}}{n+p+1}-p \frac{g^{n+p}}{n+p}+\frac{p(p-1)}{2} \frac{g^{n+p-1}}{n+p-1}-\frac{p(p-1)(p-2)}{6} \frac{g^{n+p-2}}{n+p-2}+\cdots+(-1)^{p} \frac{g^{n+1}}{n+1}
\end{aligned}
$$

above equality can be represented as

$$
f^{n+1} \sum_{l=0}^{p} \frac{(-1)^{l} C_{p}^{l}}{n+p-l+1} f^{p-l} \equiv g^{n+1} \sum_{l=0}^{p} \frac{(-1)^{l} C_{p}^{l}}{n+p-l+1} g^{p-l} .
$$

This completes the proof of the theorem.

Proof of Theorem 3. First we show that $f^{n} f^{\prime} \equiv g^{n} g^{\prime}$. Consider $k_{1}=k_{2}=k_{3}=k$ and $a=0$.We have $\bar{d}\left\{f^{n} f^{\prime}\right\}=\bar{d}\left\{g^{n} g^{\prime}\right\}=n+1, Q=1$ and $m=1$. Therefore

$$
\min \left\{D_{f}, D_{g}\right\} \geq 0 \quad, \quad \max \left\{D_{f}, D_{g}\right\}>0,
$$

where

$$
D_{f}=3(k+1)^{2} \delta_{2}(0, f)-(n+2)(k+1)(6+4 k)+(k+1)(6+4 k) \Theta(\infty, f)
$$

and

$$
D_{g}=3(k+1)^{2} \delta_{2}(0, g)-(n+2)(k+1)(6+4 k)+(k+1)(6+4 k) \Theta(\infty, g)
$$


By Theorem B, we get

$$
\begin{aligned}
f^{n} f^{\prime} & \equiv g^{n} g^{\prime} \\
\left(F^{*}\right)^{\prime} & \equiv\left(G^{*}\right)^{\prime}
\end{aligned}
$$

Then

$$
F^{*} \equiv G^{*}+c, \quad c \text { is a constant. }
$$

where

$$
F^{*}=\frac{f^{n+1}}{n+1}, \quad G^{*}=\frac{g^{n+1}}{n+1}
$$

By Lemma 2.1, we have $T\left(r, F^{*}\right)=(n+1) T(r, f)+S(r, f)$. Note that

$$
\begin{aligned}
& \overline{N_{2}}\left(r, \frac{1}{F^{*}}\right)=\overline{N_{2}}\left(r, \frac{1}{f}\right) \\
& \overline{N_{2}}\left(r, F^{*}\right)=\overline{N_{2}}(r, f)
\end{aligned}
$$

Similarly, as in the Theorem 1 , we obtain

$$
\begin{aligned}
\Theta_{2}\left(0, F^{*}\right) & =\frac{n}{n+1}+\frac{\Theta_{2}(0, f)}{n+1} \\
\Theta_{2}\left(\infty, F^{*}\right) & =\frac{n}{n+1}+\frac{\Theta_{2}(\infty, f)}{n+1} \\
\Theta_{2}\left(c, F^{*}\right) & =\Theta_{2}\left(0, G^{*}\right)
\end{aligned}
$$

and

$$
\Theta_{2}\left(0, G^{*}\right)=\frac{n}{n+1}+\frac{\Theta_{2}(0, g)}{n+1}
$$

We now show that $c=0$ in (14).Suppose that $c \neq 0$, then

$$
\begin{aligned}
\Theta_{2}\left(0, F^{*}\right)+\Theta_{2}\left(\infty, F^{*}\right)+\Theta_{2}\left(c, F^{*}\right) & =\frac{n}{n+1}+\frac{\Theta_{2}(0, f)}{n+1}+\frac{n}{n+1}+\frac{\Theta_{2}(\infty, f)}{n+1}+\frac{n}{n+1}+\frac{\Theta_{2}(0, g)}{n+1} \\
& =\frac{3 n}{n+1}+\frac{\delta_{2}(0, f)}{n+1}+\frac{\Theta(\infty, f)}{n+1}+\frac{\delta_{2}(0, g)}{n+1}
\end{aligned}
$$

Since $\min \left\{D_{f}, D_{g}\right\} \geq 0$,

$$
\begin{aligned}
& D_{f}=3(k+1)^{2} \delta_{2}(0, f)-(n+2)(k+1)(6+4 k)+(k+1)(6+4 k) \Theta(\infty, f) \geq 0 \\
& D_{f}=3(k+1)^{2} \delta_{2}(0, f)-(n+2)(k+1)(6+4 k)+(k+1)(6+4 k) \Theta(\infty, f) \geq 0
\end{aligned}
$$

Therefore

$$
\begin{aligned}
& \delta_{2}(0, f) \geq \frac{(n+2)(6+4 k)}{3(k+1)}-\frac{(6+4 k)}{3(k+1)} \Theta(\infty, f) \\
& \delta_{2}(0, g) \geq \frac{(n+2)(6+4 k)}{3(k+1)}-\frac{(6+4 k)}{3(k+1)} \Theta(\infty, g)
\end{aligned}
$$


Substituting (16) and (17) in (15), we get

$$
\begin{aligned}
& \Theta_{2}\left(0, F^{*}\right)+\Theta_{2}\left(\infty, F^{*}\right)+\Theta_{2}\left(c, F^{*}\right) \\
& \geq \frac{3 n}{n+1}+\frac{1}{n+1}\left\{\frac{(n+2)(6+4 k)}{3(k+1)}-\frac{(6+4 k)}{3(k+1)} \Theta(\infty, f)\right\}+\frac{\Theta(\infty, f)}{n+1} \\
& \quad+\frac{1}{n+1}\left\{\frac{(n+2)(6+4 k)}{3(k+1)}-\frac{(6+4 k)}{3(k+1)} \Theta(\infty, g)\right\} \\
& =\frac{3 n}{n+1}+\frac{(2 n+4)(6+4 k)}{3(k+1)(n+1)}-\frac{(k+3)}{3(k+1)(n+1)} \Theta(\infty, f)-\frac{(6+4 k)}{3(k+1)(n+1)} \Theta(\infty, g) \\
& \geq \frac{3 n}{n+1}+\frac{(2 n+4)(6+4 k)}{3(k+1)(n+1)}-\frac{(k+3)}{3(k+1)(n+1)}-\frac{(6+4 k)}{3(k+1)(n+1)} \\
& =\frac{17 n k+21 n+11 k+15}{3(k+1)(n+1)} \geq 4.666 . .
\end{aligned}
$$

Because, let

Then

$$
Q_{n}=\frac{17 n k+21 n+11 k+15}{3(k+1)(n+1)} \quad, \quad k>0
$$

$$
Q_{n}^{\prime}=\frac{18 k^{2}+36 k+18}{9(k+1)^{2}(n+1)^{2}}>0 \text { for } k>0
$$

Thus $Q_{n}$ is an increasing function and

$$
Q_{n} \quad \text { at } \quad n=1=\frac{28 k+36}{6 k+6} \quad, \quad \lim _{k \rightarrow \infty}\left\{\frac{28 k+36}{6 k+6}\right\}=\frac{28}{6}=4.6666 \ldots . .
$$

This shows that $Q_{n}$ always exceeds the value 4.666...which contradicts Lemma 2.2. Hence $c=0$. Therefore

$$
\begin{aligned}
F^{*} & \equiv G^{*} \\
\frac{f^{n+1}}{n+1} & =\frac{g^{n+1}}{n+1}
\end{aligned}
$$

Let $h=\frac{f}{g}$. If $h \equiv 1$ then $f \equiv g$. Suppose $h \not \equiv 1$, then $h^{n+1}-1=0$. This completes the proof of the theorem.

\section{References}

[1] Anupama J. Patil, Nevanlinna theory- investigations and some applications. Ph.D Thesis, Karnatak University, Dharwad. Karnataka-State, India, October 2007.

[2] A. Z. Mokhon'ko, On the Nevanlinna characteristics of some meromorphic functions. Theory of functions, Functional Analysis and their Applications, 14 (1971), 83-87.

[3] W. Doeringer, Exceptional values of differential polynomials, Pacific J. Math., 98(1982), 55-62.

[4] G. Frank and M. Reinders ,A unique range set for meromorphic functions with 11 elements, Complex Variables, 37 (1998), 185-193. 
[5] H. X. Yi, The multiple values of meromorphic functions and uniqueness, Chin. Ann. Math. (A), 10(1989), 421427.

[6] L. Yang, Value Distribution Theory. Springer-Verlag, Berlin, 1993.

[7] S. S. Bhoosnurmath and Anupama J. Patil, On the growth and value distribution of meromorphic functions and their differential polynomials. Journal of the Indian Math. Soc., 74(2007), 167-184.

[8] W. K. Hayman, Meromorphic Functions. Claredon Press, Oxford, 1964.

${ }^{1}$ Department of Mathematics, Karnatak University, Dharwad-580003, India.

E-mail: ssbmath@gmail.com

${ }^{2}$ Department of Mathematics, Karnatak University, Dharwad-580003, India.

E-mail: vlpujari@gmail.com

${ }^{3}$ Department of Mathematics, Bristol University, UK.

E-mail: ajpatil81@gmail.com 